\section{PTU-47 THE ROLE OF CROSS-SECTIONAL IMAGING IN THE MANAGEMENT OF PATIENTS WITH COMPLEX COLORECTAL POLYPS}

Clare Westwood*, losif Beintaris, John Jacob. University Hospital North Tees And Hartlepool Nhs Trust, Stockton, UK

\subsection{6/gutjnl-2021-BSG.120}

Introduction Current guidelines state patients diagnosed with colorectal cancer should undergo computed tomography scanning of chest, abdomen, and pelvis (CT CAP) for staging, plus MRI of the pelvis and rectum for rectal cancers. Requests for scans are usually made at endoscopy when malignancy is suspected. Colorectal cancer is usually an obvious diagnosis; however, large colorectal polyps also harbour an increased risk of malignancy. There are currently no firm guidelines as to when we should perform radiological staging investigations following identification of a non-overtly malignant colorectal polyp. Currently, common practice is that patients who are found to have a colorectal polyp felt to harbour a high risk of covert cancer at endoscopic assessment, have CT CAP +/- MRI requested at the time of endoscopy, in case a cancer is eventually histologically confirmed. This practice is reinforced by tight timelines of cancer ('two week wait') pathways.

Performing radiological investigation is not without risk. CT holds risks of ionising radiation exposure and should only be used in patients who would benefit from the information the investigation provides. It is important that resources such as CT and MRI are used efficiently.

Methods The aim of this retrospective review was to analyse the use of cross-sectional imaging following a diagnosis of complex colorectal polyp to determine if results influenced clinical decision-making and onward patient management. All cases discussed at the complex polyp MDT within North Tees and Hartlepool NHS Trust, since its inception in January 2020 were included.

Patients were identified and reviewed by accessing electronic clinical notes and results software. Information on polyp assessment at time of endoscopy was reviewed, including any increased cancer risk as assessed by the endoscopist. Electronic records were accessed by two clinicians independently, who reviewed post polypectomy histology to determine if the radiological investigation had assisted and/or altered the patient's management.

Results From 23rd January 2020 to 11th February 2021, 151 cases were discussed. Repeat discussions were excluded. Radiological imaging was performed in 47; 11 were excluded as the investigation was not in relation to the complex polyp.

36 records were analysed as part of this review; polyp diameter ranged from 1 to $7 \mathrm{~cm}$. Radiological investigations consisted of CT CAP only $(n=27)$, CT CAP with MRI $(n=7)$ and MRI only $(n=2)$. In $13 / 36$ cases, imaging did not alter subsequent management of the patient; in these cases, endoscopic image review at the complex polyp MDT meeting was the key influencer on treatment options. In 23/36 cases, cross sectional imaging did, at least partially, inform clinical management of the patient; however, in only 2 of these cases imaging altered the overall management plan:

Conclusions From this retrospective review, we identified that in patients with complex colorectal polyps, radiological assessment is secondary to endoscopic assessment in treatment decision making. High-risk polyp features can be identified from good quality endoscopic assessment with image enhancement and magnification. Cross sectional imaging should be requested in cases with overt evidence of malignancy for the purposes of staging. Good endoscopic photo documentation of any lesion is key to facilitate MDT discussion in the planning of surgical vs endoscopic resection to ensure patients undergo the right procedure first time.

\section{PTU-48 OUTCOMES OF OESOPHAGO-GASTRO-DUODENOSCOPY (OGD) IN THE INVESTIGATION OF INPATIENTS WITH ANAEMIA: A RETROSPECTIVE COHORT STUDY}

'Alice Lagnado*, ${ }^{1}$ Charles Broome, 'Shwan Karim, 'Salman Ahmed, ${ }^{2}$ Christopher Wadsworth. ${ }^{1}$ Hammersmith Hospital, London, UK; ${ }^{2}$ Hammersmith Hospital, London, UK

\subsection{6/gutjil-2021-BSG.121}

Introduction Iron deficiency anaemia (IDA) is a common diagnosis affecting $2-5 \%$ of adult men and post-menopausal women. Referrals for inpatient investigation of IDA represent a significant workload burden. However, performing inpatient endoscopy can delay discharge and carries a well reported morbidity and mortality. We felt a significant proportion of these investigations had low yield and therefore, we undertook a study to determine the outcomes of inpatient OGDs and if there were any factors that were associated with an increased likelihood of positive findings.

Methods This was a single-centre retrospective cohort study. Data on sequential patients who had an inpatient OGD over a three-year period was collected. Patients were excluded from the study if they had evidence of overt GI haemorrhage or haemodynamic instability. Multivariate logistic regression used to analyse the primary outcomes and the covariates. The primary outcome of the study was whether patients had a positive finding on OGD that may have contributed to their anaemia as defined by the British Society of Gastroenterology (BSG) guidelines (1). The variables studied were age, referring speciality and prescription of antiplatelets or anticoagulants prior to OGD. Secondary outcomes included whether patients' management was changed by having an inpatient OGD.

Results 147 patients had inpatient OGDs for IDA, median age of 69 (27-92). Referrals were mainly made by Cardiology (37.9\%) and Renal (35.2\%) physicians. 83 patients were taking one or more antiplatelet medications and 25 were taking anticoagulants, of which 13 were on both.

The different OGD findings, and numbers of each are highlighted in the table below. Only $22(15 \%)$ had one or more possible causes for IDA identified, of which $11(7.5 \%)$ resulted in change of their clinical management; 1 had therapy during the procedure (banding), 4 had a previously unknown malignancy detected, 4 had their anti-platelet medication either stopped or not started, one was commenced on a b-blocker for varices, another was started on the Hong-Kong PPI infusion protocol.

Multivariate logistic regression analysis was carried out on the data using $\mathrm{R}$ v. 4.0.2. The analysis of the independent variables (age $[p=0.3912]$, anticoagulation $[p=0.4788$ ], antiplatelet $[p=0.5829]$ and referring speciality) showed no statistically significant predictive value. Therefore, none of the above factors could be used to determine the likelihood of inpatient OGD changing management.

Conclusion Limited by the retrospective nature of this study, the findings show only a small proportion of inpatient OGDs $(15 \%)$ find a potential cause of IDA. In addition to this, the 